\title{
An Optimized Approach for Selecting an Optimal Number of Cell Site Locations in Cellular Networks
}

\author{
Williamjeet Singh \\ Phd Scholar UCoE, \\ Punjabi University \\ Patiala, Punjab
}

\author{
Jyotsna Sengupta \\ Head of Department DCS, \\ Punjabi University \\ Patiala, Punjab
}

\begin{abstract}
Wireless communication has seen gigantic advancement all the way through past years. The maturity of newer generations of technology and boost up in user mobility has created the need and demand for wireless networks that has triggered considerable technological advances as well as the investigation of optimization algorithms to support design and planning decisions. Wireless system providers will be vital to enlarge their infrastructure rapidly in order to meet this swift escalation in wireless data demand. Demand for cheaper and better wireless communication services from customers are the key factors to optimally design the cell geometry and select the minimum number of cell sites to provide maximum possible coverage. In this paper, we consider how to optimally determine the cell site locations such that, number of base stations $(\mathrm{N})$ is minimum while coverage is maximum so that best possible service is possible with minimum infrastructural costs. An optimized algorithm is presented here that determines the optimal locations of base stations without performing an exhaustive search. The algorithm simulates the network, uses the function to rank the cells and then applies screening criteria that removes the cell with the and repeats the process until $k$ cells are removed.
\end{abstract}

\section{General Terms}

Algorithms, Cellular Networks, Design and Planning.

\section{Keywords}

Cell site, Network design, Optimization, Coverage, Base Station, Rank, Screening Criteria.

\section{INTRODUCTION}

Cell planning is regarded as the process of selecting the locations of base stations in the wireless network. Cell planning is a fundamental and challenging part of cellular network design process $[1,14]$. The automatic techniques that lend a helping hand to locate the optimal number of cell sites in a specified area are indispensable due to non-uniform user locations and traffic fluctuations. The goal of planning primarily focuses on the selection of sites and equipment to maximize the coverage. In cell planning for mobile communication systems, numerous baseline issues like traffic demand to cover a specific region, availability of base station sites, available channel capacity at each base station and the service quality at various potential traffic demand areas (TDAs) are needed to be considered [13]. The exact size of the cells actually vary significantly due to several factors, including the topography of the land, the anticipated number of calls in a particular area, the number of obstacles, and the traffic pattern of the mobile users. For cellular wireless systems, mobile communication is facilitated by base stations which have an appropriate spatial distribution. The problem discussed in this paper is to determine the number of base stations and location and capacity of each base station to cover increased traffic demand. Selected sites must be configured to provide adequate service coverage and capacity while adhering to constraints involving regions which can be served by more than one antenna. Most of the research in the optimization of the radio coverage in cellular system is restricted to the selection of base station locations. Optimal coverage with minimum number of base stations is essentially a resource allocation/optimization problem. The overall goal of cell planning is to select a sufficient subset of base station sites at the appropriate spatial density from a set of candidate sites to meet network design objectives. Various forms of inputs and limitations from the customer in terms of spectrum availability, network dimensions, frequency planning, network growth, local wireless regulations and finally the RF environment itself plays an important role in coverage planning. The approach for the coverage plan needs to be well defined, since it requires accommodating various phases of network growth across time without any compromise on service quality goal.

In the increasingly competitive wireless industry, optimization approach offers the means to achieve good quality solution consistently. While deploying a network, the optimization phase is followed immediately after the new frequency plan is introduced. Several teams of field personnel undertake extensive drive testing around each site making a number of calls, concentrating on testing and the handovers between each cell. Each call is investigated and any identified or potential problems resolved by classical faultreasoning/resolution methods. Drive-testing methodology is used by most network operators so as to identify the areas within the network for improvement through optimization. This method of network performance measurement is very important for comparing the performance of network under test with competitor's network.

The placement of base stations becomes a difficult for system designers, the reason being the frequency channels become increasingly congested and propagation environments become more complex. Suboptimal placement of base stations will result in not only expensive deployment costs, but a reduction in spectrum efficiency (due to interference) which could be devastating to a service provider considering the cost of the spectrum license. In order to cope with the need of rapid wireless systems deployment, a significant amount of effort has been expended on research to develop advance wireless planning techniques over the past few years. When the cellular concept was first proposed, base station locations were usually selected according to a regular reuse pattern [4, 5]. Extensive tuning is often required in order to reach a required standard of performance. Operators want to reuse as 
many site locations in their networks to cut network costs. Over the years, these wireless planning techniques have evolved within the industry. Service providers are becoming more aware of the importance of resource management and a significant amount of work has been published in the literature with regard to advanced wireless planning techniques. In general, the wireless planning methodology has shifted from a manual procedure to a computer-aided semiautomated procedure.

\section{NEED OF THE STUDY}

In any wireless network planning problem, the radio model is a key component. Achieving the maximum capacity along with maintaining an acceptable grade of service and good speech quality is the main issue for the network planning. Mobile cellular networks were initially designed for voice service but nowadays broadband multimedia services have been introduced into mobile wireless networks leading to increased traffic demand. With an intention to enhance network capacity and satisfy user demand of broadband services; reducing the cell size is one of the most effective approaches to improve the spatial reuse of radio resources. Most cellular phone users have had unpleasant experiences, such as disconnections or unstable and weak connections during conversations due to the inadequate capacities of the cell site to handle call traffic in a cellular telecommunication. From the viewpoint of end users, full coverage is particularly desirable although 100\%-coverage is not yet reached because operators often have many constraints when installing large base stations and antennas. This generally results in potential coverage holes, bared and dead zones. Every cellular network deployment requires planning and optimization in order to provide adequate coverage, capacity and quality of service (QoS). Cell planning is a fundamental and challenging part of cellular network design process. Due to non-uniform users' locations and traffic fluctuations, the automatic techniques that locate base stations in a specified area are necessary. The location of base stations (BS) is of paramount importance for the performance of cellular radio networks. Hence, in the planning phase of a network there should be an automated tool available which suggests optimum location of base stations

\section{RESEARCH OBJECTIVES}

This study is aimed at designing an efficient algorithm for optimally locating the base-stations and the assignment of transmission ranges in any given service area. In achieving the research objectives, this study intends to answer the following three questions:

(i) Is there any promising technique to position the basestations such that every point in the region is covered by at least one base-station?

(ii) Is there any approach to optimize the number of base stations in any given area?

(iii) How can we simultaneously assign and minimize the transmission range for each base station?

(iv) What are the plausible benefits of this research with respect to society?

\section{PROBLEM STATEMENT}

The problem of optimization of the base station locations can thus be stated as: Given an area to cover, identify the optimal cell geometry for locations of the base stations such that number of base stations is minimal while the coverage is maximal so that best possible service is possible with minimum infrastructural costs. Our problem is to choose a set of $k$ cells to delete among $N$ possible candidate cells so as to maximize the performance of the network. Given the combinations of possible cells to choose it is clear that this is a combinatorial optimization problem [2] with possible solutions. In fact, if we place no restrictions on the behavior of the network performance function, then cell deletion is provably EXPTIME-hard [7].

\section{RELATED WORK}

Radio network design report provides with the coverage areas with number of macro cellular sites planned with their respective configuration that allows the planner to have a good idea of the total number of sites expected in different areas in different phases of implementation. Furthermore, the link budget calculation, frequency planning and coverage planning are made to change with phases of the network. Consider a case where the customer plans to have 100 sites in its final phase and wants to implement them across 3 phases. He recommends that in the first phase he want to implement 50 sites, in second phase 30 sites and in final or third phase 20 sites. Here the planner makes a plan as per 100 sites and does all the necessary calculations to achieve the desired objective in terms of coverage and quality of service. Having done this he goes about planning for second phase where he plans for 80 sites. In this case he does re-calculation of the site parameters, re-defines the link budget, frequency plan, etc. He does similar calculation for phase one with 50 sites and repredicts the results. It is to be noted at this point that the customer sets his bench marks for individual phases and the planner needs to plan the network to meet this benchmark. Cell planning is a crucial part of designing a mobile communication network. Although, there are many proposed algorithms for this purpose, the problem has been remained challenging in non-uniform traffic situations. Several parameters exist for cellular network design. The most effective parameters are the number of cells and the coverage area of each cell supported by each cell. The classic method for this purpose is Genetic Algorithm (GA) and has been studied in different papers $[10,11]$. Although, this algorithm is very useful for many practical optimization problems, however this method have some deficiencies for this special task, i.e. cell planning in wireless communication networks. The main deficiency of GA is that it is not applicable to large number of cells due to its computational complexity. Previous work on the site selection problem focused on choosing cell sites using simulated annealing [11, 12], evolutionary algorithms [8], integer linear programming [10], and the greedy algorithm [6]. None of the mentioned previous work includes all the three vital parameters (in network planning): maximizing the network coverage, maintaining signal to interference noise ratio for satisfactory performance and reducing the cost of the network.

\section{PROPOSED WORK}

The structure of the proposed method lets several parameters be applied in cellular mobile communication network design simultaneously like capacity, signal to noise (SIR) ratio and performance. In continuation with our previous work [15], we are going to optimize an algorithm one step further so as to provide more efficient results while choosing the best cell site locations. The research work is inspired by the concept provided by David Abusch-Magder [3] to focus on the related problem of optimally choosing the cell sites so as to provide maximum coverage while maintaining the performance or quality parameter. David is concentrating on the concept of cell site removal during the technology upgrade and has 
provided a general algorithm without considering any parameter whereas the novelty of this paper is to utilize the algorithm in planning phase where we are given number of candidate sites and we have to eliminate or neglect certain sites for economic reasons but still coverage and quality parameter like performance are top priority. The possible count of cell sites is provided as input to the algorithm.

\section{OPTIMIZED ALGORITHM}

At the core of our algorithm is a function which ranks the cells based on signal to interference noise ratio. The algorithm simulates the network, uses the function to rank the cells, removes the cell with the highest ranking, and repeats the process until $k$ cells are removed. If a cell were to be removed the chance that a mobile would be left uncovered is correlated to each mobile's hand-off state; we exploit this correlation when constructing our sample ranking function. Furthermore, we have to show a modification to the proposed algorithm, which allows for the screening of the rejected candidate before the final rejection is allowed. An additional function is to be designed that will further determine whether the proposed network has acceptable performance; if it does not, the algorithm rejects the highest ranked candidate and seeks the candidate with the highest ranking that passes the screening function. The screening criteria, like the ranking function, will depend on the provider's goals (like coverage) and on the data provided as an input. Mobiles that are in handoff with two or more cells (soft hand-off) are unlikely to be abandoned if one of those cells is removed from the network configuration. The choice of the ranking function will depend on the three parameters: capacity, signal to interference noise (SINR) ratio and performance. This algorithm may be used independently of the models adopted to simulate the network, as long as an appropriate ranking function can be defined. Following are the algorithmic steps for the approach that we have followed.

Step 1: Consider a network that constitutes a set of $M$ cells $C$ $=\left\{C_{i} \mid 1 \leq i \leq M\right\}$, for which the three parameters: capacity, signal to noise (SIR) ratio and performance are calculated respectively.

Step 1a: Capacity of any cell is given by:

capacity $=($ calls $/ 3600) *$ htime;

where, calls $=55+\operatorname{random}(5)$;

htime $=360+$ random $(60)$; // sixty calls/minute

Step $1 b$ : SINR of any cell is given by:

$\operatorname{sir} 1=1 /(($ users- 1$)+($ noise/signal $))$;

where, users $=55+\operatorname{random}(5)$;

noise $=10+\operatorname{random}(5) ; \operatorname{signal}=90+\operatorname{random}(5)$;

Step 1c: Performance of any cell is given by:

$\mathrm{P}_{\mathrm{i}}=$ (tempcap + tempsir $) / 2$;

where, tempcap $=(\mathrm{c} 1 / 7) * 100$;

tempsir $=100-\left(((\mathrm{s} 1-0.0169) / \mathrm{s} 1)^{*} 100\right)$;

and the overall performance of network is given by the average of each individual cell performance.

Step 2: For each cell, we identify the base station(s) that cover the given mobile node. It may happen that one mobile node is covered by more than one base station. But the intention is never to lead any mobile station isolated.
Step 3: We are provided with a ranking function: $C \mapsto \mathbb{R}^{|D|}$ based on the data gathered based on SIR factor.

Step 4: Out of total M cells, a set of first $N$ cells, $\mathrm{D}=\left\{C_{i} \mid 1\right.$ $\leq i \leq N\} \subseteq C$ are candidates for removal (where, isolation = 0 ; isolation is the function that updates the isolation array of each base station).

Step 5: Define a set $\mathrm{R} \leftarrow \varnothing$ that contains set of cells to remove. We must choose a set $R \subset D$ containing $k$ of the $N$ candidate cells that does not allow the network performance to degrade much.

Step 6: Define another set $D_{0}=\left\{C_{i} \mid 1 \leq i \leq N\right\}$ that contains set of cells which are candidates for removal, i.e. the cells that are not at all covering any mobile station.

Step 7: A variable $1 \leftarrow|\mathrm{R}|=0$, keep track of the number of cells deleted so far.

Step 8: Repeat the following steps while $(1<\mathrm{k})$

Step 8a: Calculate the ranking of all candidate cells based on a SIR with cells in $R_{l}$ removed. $\left(\mathrm{r}_{1,1}, \mathrm{r}_{1,2}, \mathrm{r}_{1,3}, \ldots \ldots \ldots, \mathrm{r}_{1, \mathrm{~N}}\right) \leftarrow$ score $\left(\mathrm{C}-\mathrm{R}_{\mathrm{l}}\right)$

Step $8 b: \mathrm{F} \leftarrow \varnothing$, initialize list of cells that have failed to pass screening criteria

do

if $\mathrm{C}_{\mathrm{j}}$ just failed screening criteria

then $F_{1} \leftarrow F_{1} \cup\left\{C_{j}\right\}$, list of cells that failed in this round

$\mathrm{j} \leftarrow \operatorname{argmax}_{\mathrm{i}} \in\{\mathrm{m} \mid \mathrm{Cm} \in(\mathrm{Dl}-\mathrm{Fl})\}\left(\mathrm{r}_{\mathrm{l}, \mathrm{i}}\right)$

choose cell $\mathrm{C}_{\mathrm{j}} \in\left(\mathrm{D}_{1}-\mathrm{F}_{\mathrm{l}}\right)$ which has the

highest $\mathrm{r}_{\mathrm{l}, \mathrm{j}}$, is a candidate, and has not

failed the screening criteria

until $\left(\operatorname{screen}\left(\mathrm{R}_{1}, \mathrm{C}_{\mathrm{j}}\right)=1\right.$ or no cells remain)

if no cells remains

then choose cell $\mathrm{j} \leftarrow \operatorname{argmax}_{\mathrm{i}} \in\{\mathrm{m} \mid \mathrm{Cm} \in \mathrm{Dl}\}\left(\mathrm{r}_{\mathrm{l}, \mathrm{i}}\right), \mathrm{C}_{\mathrm{j}} \in \mathrm{D}_{1}$ which has the highest score even though it failed screening criteria.

At this stage we are left with only those BSs which have highest rank and cleared the screening criteria.

Step 8c: $\mathrm{R}_{1+1} \leftarrow \mathrm{R}_{\mathrm{l}} \cup\left\{\mathrm{C}_{\mathrm{j}}\right\}$, this is the set of cells removed in the $(1+1)^{\text {st }}$ step

Step 8d: $\mathrm{D}_{1+1} \leftarrow \mathrm{D}_{1}-\left\{\mathrm{C}_{\mathrm{j}}\right\}$, remove $\mathrm{C}_{\mathrm{j}}$ from candidate list

Step $8 e: 1 \leftarrow\left|\mathrm{R}_{1+1}\right|=1+1$, update count of cells already deleted

endWhile

Output: The suggested deletions from 0 to $\mathrm{k}\left\{\mathrm{R}_{1} \mid 0 \leq 1 \leq \mathrm{k}\right\}$ and all the ranks $\left\{\mathrm{r}_{1, \mathrm{i}} \mid 0 \leq 1 \leq \mathrm{k}\right\}, \quad \mathrm{C}_{\mathrm{i}} \in \mathrm{D}_{\mathrm{l}}$ associated with each deletion list.

\section{DETAILED WORKING OF AN ALGORITHM}

Fig 1 described next clearly depicts the flow of how to select an optimal number of cell site locations for cellular networks. A step by step execution is described thereafter. We are also providing the snapshots of the actual results obtained during the implementation phase that gives clear idea of the work done. 


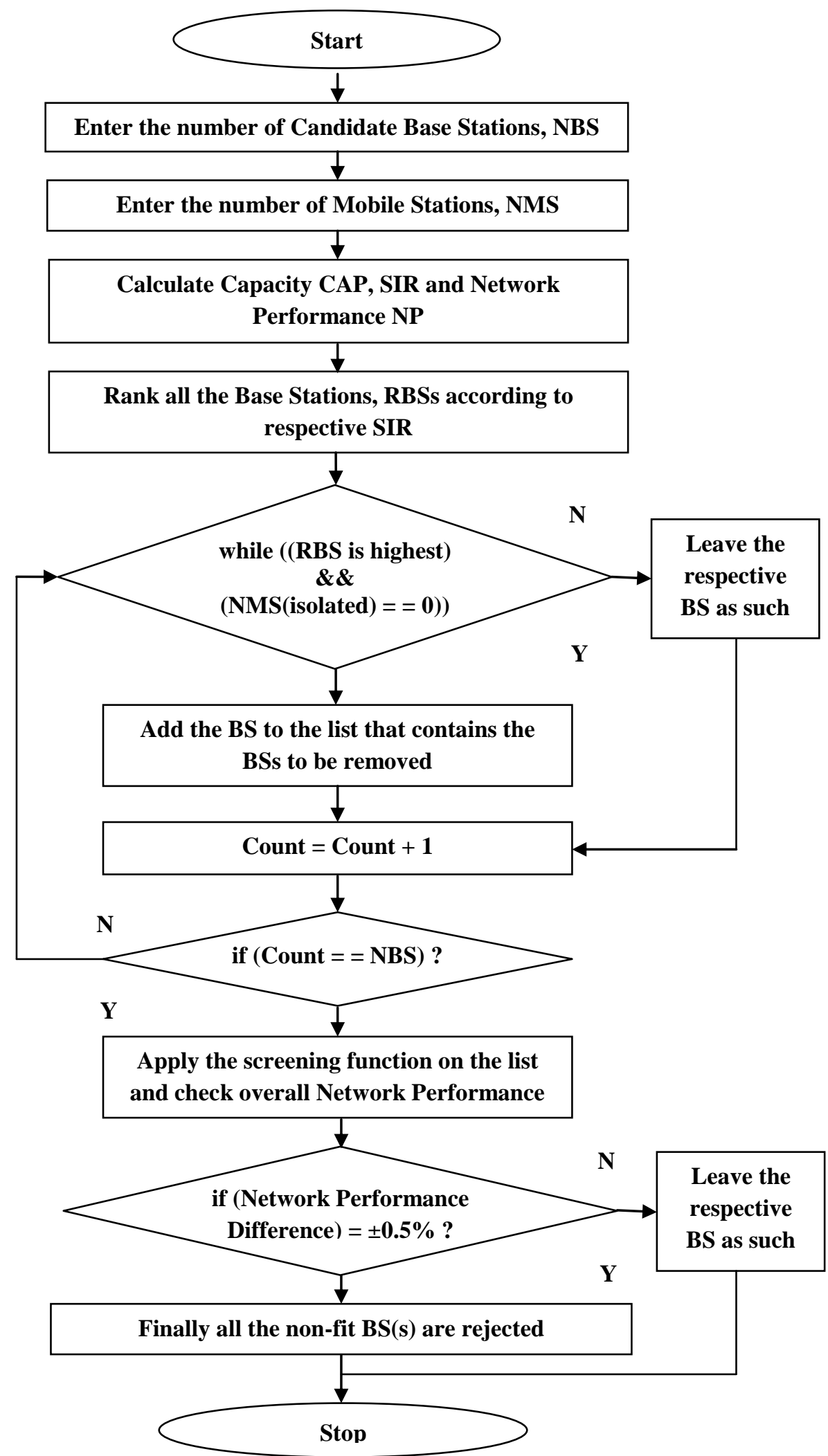

Fig 1: Flowchart for Selecting an Optimal Number of Cell Site Locations in Cellular Networks 
Step 1: The two inputs are taken initially in the form of maximum (candidate) number of base stations NBSs ( 8 here) as well as the number of mobile stations NMS to be served ( 8 here). Based on this data, the capacity, SINR and performance of the individual MS are calculated.

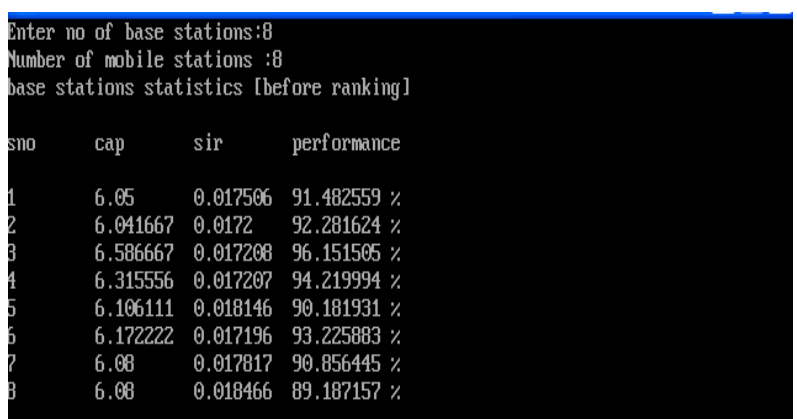

Fig 2: Input of NBS, NMS

Step 2: All the 8 MSs are checked, whether they are served by one (or more) BS(s) or they are left isolated. Then the raking of all BSs is done based on rank function (signal to interference noise ratio).

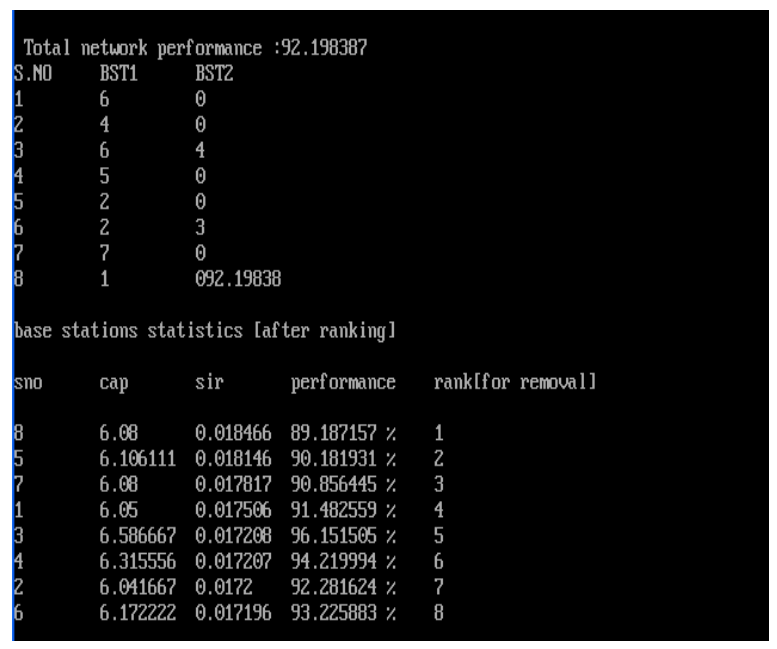

Fig 3: Implementation of Rank Function on BSs

Step 3: Now at this stage we have obtained the list of BSs (3 and 8) that can be removed without leaving any MS isolated.

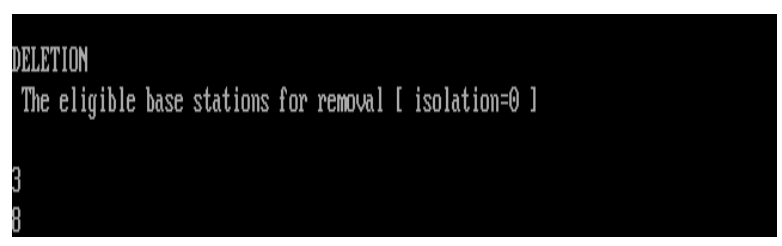

Fig 4: Cell Site Optimization Phase

Step 4: Then the optimization phase starts, where we further screen the above list of BSs to be removed. Here we apply other criteria to check whether the removal of these BSs is adversely affecting the performance or not. It may happen that due to the rejected BSs, the load on other BSs increases (even beyond their capacity) and the overall scenario starts providing inefficient results. So, to avoid this, we have maintained a check that will validate the overall performance after each BS (from the list) is deleted one by one, rather than ignoring the whole list at one go. It is clear from the following simulation snapshot that the overall network performance is $92.19838 \%$ and if we try to remove the BS-3 then this network performance increases to $91.63365 \%$. Although it sounds good that the performance has increased but still we have not allowed the BS-3 to be removed. The reason is same as mentioned above that the rejection of BS-3 causes unnecessary load on other BSs. Also, we have maintained certain level of performance difference of $\pm 0.5 \%$ only. It means the rejection or selection of a BS depends upon whether this difference is within the limit of $\pm 0.5 \%$ (removed from list of BSs to be deleted) or not (remain in the list of mandatory BSs). This is the reason why BS-8 is rejected (network performance difference $=0.43 \%$ ) and BS-3 is not rejected (network performance difference $=0.56 \%$ ).

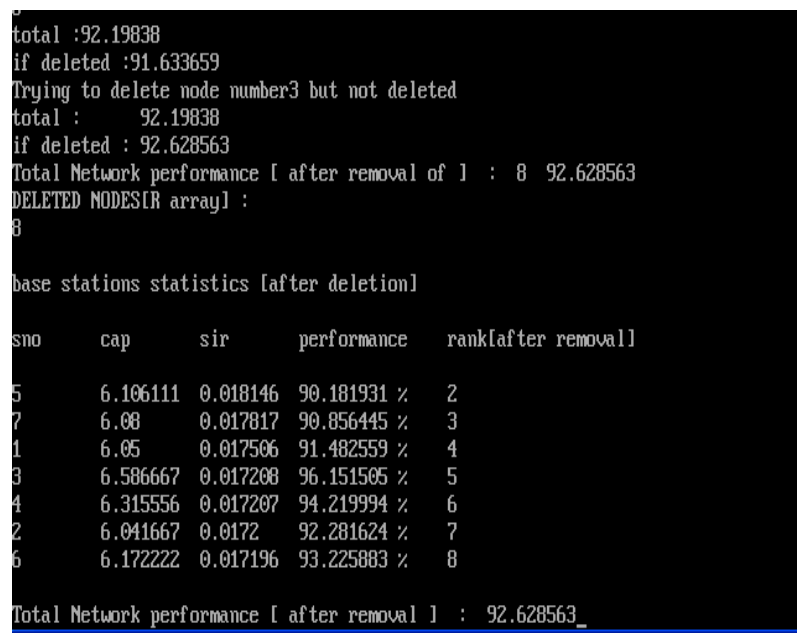

\section{Fig 5: Cell Site Optimization Phase}

Step 5: Finally we obtain 7 mandatory cell sites (BS-8 is candidate for deletion out of BS-3 and 8) that still cover all the 8 MSs with an improved network performance to $92.62856 \%$ from $92.19838 \%$

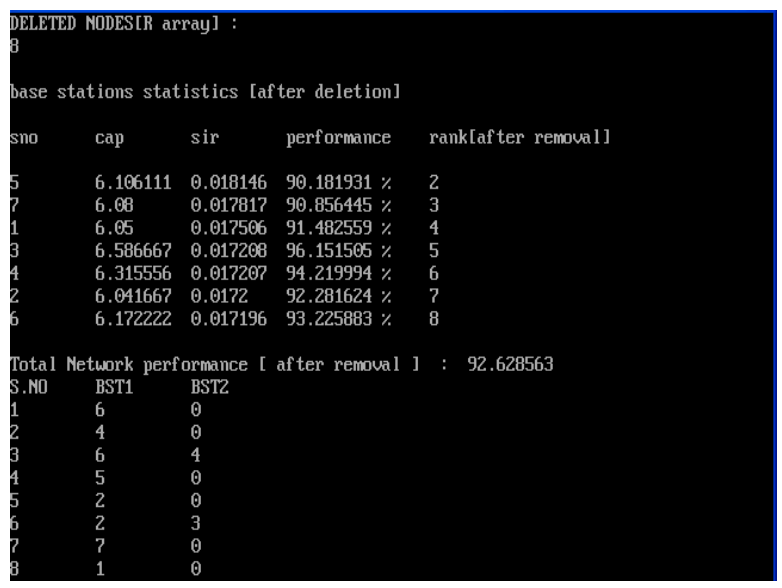

Fig 6: Resultant List of Mandatory Cell Sites

\section{ANALYSIS OF THE RESULTS}

The difference between our earlier implemented approach and the newly applied research is shown in Fig 7, depicting the inclusion of screening method that provides one step further to filter the candidate BSs chosen for rejection. This additional function has been designed to verify whether the 
proposed network has acceptable performance; if it does not, the algorithm rejects the highest ranked candidate and seeks the candidate with the highest ranking that passes the screening function. The screening criteria, like the ranking function, will depend on the provider's goals (like coverage) and on the data provided as an input. Fig 7 indicates the increase as well as smooth network performance after applying screening method. Both the rank and screening methods have been applied on varying number of base stations $(10,12,14,16,18$ and 20) and mobile stations (4, 6, $8,10,12,14,16,18$, and 20).

\section{CONCLUSION}

To find good network designs we have developed an algorithm that will guide us through the vast number of possible solutions. Like other optimization problems we were seeking algorithm whose results are optimal as well as reliable to produce good solutions in a reasonable amount of time. We have successfully implemented the task of finding the most suitable set of base stations ( 7 here) among the predefined sites ( 8 here), taking into account the following objectives of maximizing the network coverage, maintaining a certain signal to interference ratio for satisfactory performance (an increase from $92.19838 \%$ to $92.62856 \%$ ) as well as minimization of the cost of the network by using fewer sites.

\section{REFERENCES}

[1] A.R.S. Bahai and H.Aghvami, "Network planning and optimization in the third generation wireless networks," $3 G$ mobile Communication Technologies, Conference Publication No. 471, IEEE 2000.

[2] C. H. Papadimitriou and K. Steiglitz, Combinatorial Optimization: Algorithms and Complexity. Mineola, N.Y.: Dover Publications, 1998.

[3] D. Abusch-Magder, "Novel Algorithms for Reducing Cell Sites During a technology Upgrade and Network Overlay," in Proceedings of WCNC 2005 by IEEE Communication Society, pp. 1726-1732.

[4] E. Amaldi, A. Capone, and F. Malucelli, "Planning UMTS Base Station Location: Optimization Models with Power Control and Algorithms," IEEE Transactions on Wireless Communications, vol. 2, pp. 939-952, 2003.

[5] Ilker Dermirkol, Cem Ersoy, M. Ufuk Caglayan and Hakan Delic, "Location Area Planning and Cell-toSwitch Assignment in Cellular network", IEEE INFOCOM 2001, Anchorage, Alaska, USA, April 2001.
[6] K. Tutschku, "Demand-Based Radio Network Planning of Cellular Mobile Communication Systems," in Proceedings of the 17th Annual INFOCOM, San Francisco, CA, 1998, pp. 1054 - 1061.

[7] M. R. Garey and D. S. Johnson, Computers and Intractability: A Guide to the Theory of NPCompleteness. New York, NY: W.H. Freeman, 1979.

[8] N. Weicker, G. Szabo, K. Weicker, and P. Widmayer, "Evolutionary Multiobjective Optimization for Base Station Transmitter Placement with Frequency Assignment," IEEE Transactions on Evolutionary Computation, vol. 7, pp. 189-203, 2003.

[9] R. Bose, "A Smart Technique for Determining BaseStation Locations in an Urban Environment," IEEE Transactions on Vehicular Technology, vol. 50, pp. 4347, 2001.

[10] R. Mathar and T. Niessen, "Optimum Positioning of Base Stations for Cellular Radio Networks," Wireless Networks, vol. 6, pp. 421-428, 2000.

[11] S. Hurley, "Planning Effective Cellular Mobile Radio Networks," IEEE Transactions on Vehicular Technology, vol. 51, pp. 243-253, 2002.

[12] S. U. G. Thiel, P.; Ibbetson and L.J.; Lister, D., "An Automated UMTS Site Selection Tool," in Proceedings of Third International Conference on $3 G$ Mobile Communication Technologies, London, UK, 2002, pp. 69-73.

[13] Williamjeet Singh and Jyotsna Sengupta, "Optimal Cell Site Planning in Varying Geographic Regions for Cellular Networks", International Journal of Computer Applications in Engineering, Technology and Sciences (IJ-CA-ETS), Volume 3: Issue 1, Oct 2010 - March 2011.

[14] Williamjeet Singh and Jyotsna Sengupta, "Performance Estimation of a Cell Site in Cellular Networks Using WiMax Technology", International Journal of Information Sciences and Application, Volume 2: Number 3, November, 2010

[15] Williamjeet Singh and Jyotsna Sengupta, "Performance Estimation of a Cell Site in Cellular Networks Using WiMax Technology", International Journal of Theoretical and Applied Information Technology, Volume 28: Number 1, June, 2011 


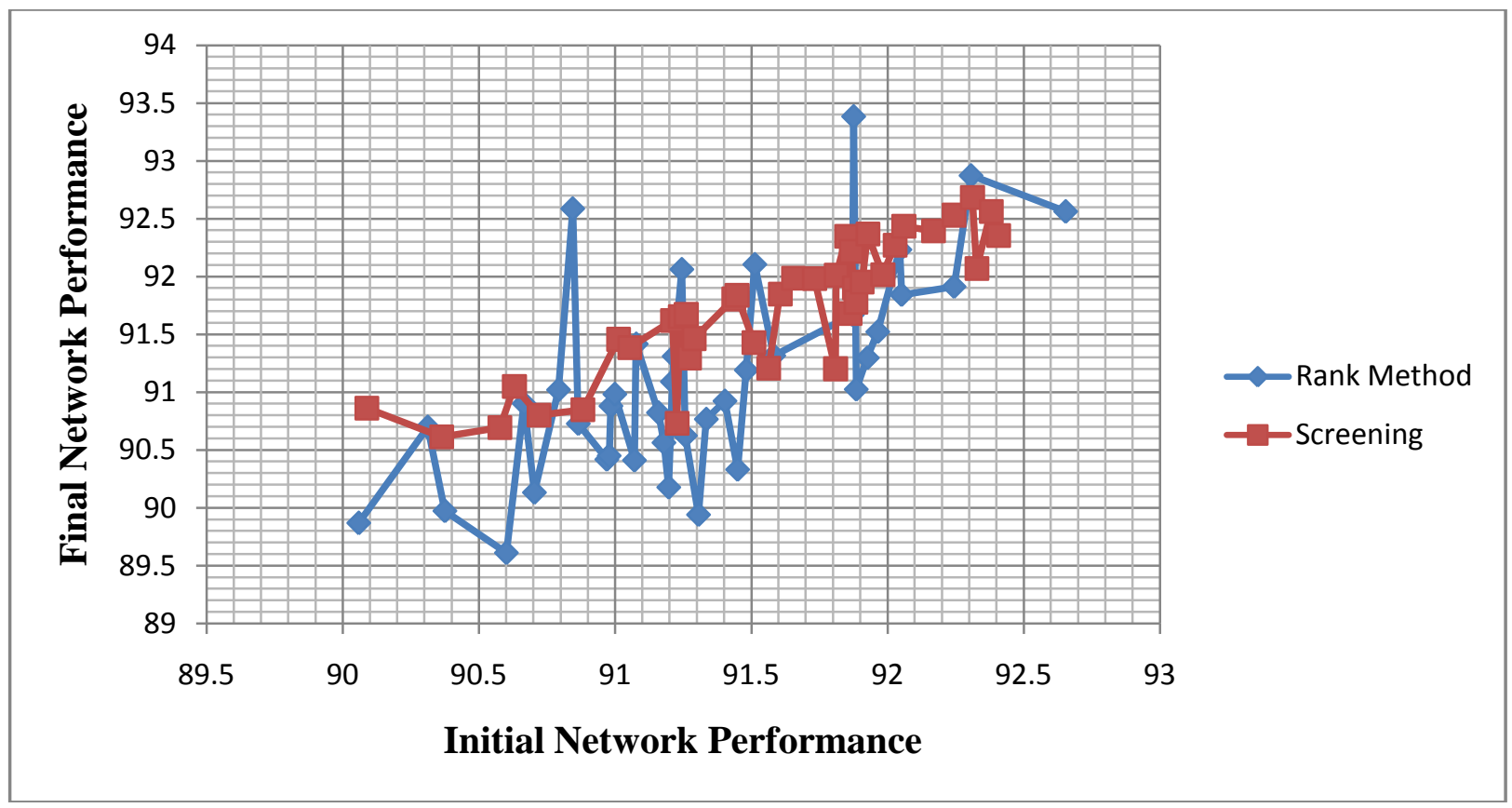

Fig 7: Comparative Analysis of the Network Performance for Rank Method and Screening Method 